Published in final edited form as:

J Magn Reson Imaging. 2011 March 1; 33(3): 527-534. doi:10.1002/jmri.22504.

\title{
MRI in patients with inflammatory bowel disease
}

\author{
Michael S. Gee, MD, PhD ${ }^{1,2}$ and Mukesh G. Harisinghani, MD ${ }^{1}$ \\ ${ }^{1}$ Division of Abdominal Imaging and Interventional Radiology, Dept. of Radiology, Massachusetts \\ General Hospital, Harvard Medical School, Boston, MA \\ 2 Division of Pediatric Imaging, Dept. of Radiology, Massachusetts General Hospital, Harvard \\ Medical School, Boston, MA
}

\begin{abstract}
Inflammatory bowel disease (IBD) affects approximately 1.4 million people in North America and, because of its typical early age of onset and episodic disease course, IBD patients often undergo numerous imaging studies over the course of their lifetimes. CT has become the standard imaging modality for assessment of IBD patients because of its widespread availability, rapid image acquisition, and ability to evaluate intraluminal and extraluminal disease. However, repetitive $\mathrm{CT}$ imaging has been associated with a significant ionizing radiation risk to patients, making MRI an appealing alternative IBD imaging modality. Pelvic MRI is currently the imaging gold standard for detecting perianal disease, while recent studies indicate that MRI bowel-directed techniques (enteroclysis, enterography, colonography) can accurately evaluate bowel inflammation in IBD. With recent technical innovations leading to faster and higher resolution body MRI, the role of MRI in IBD evaluation is likely to continue to expand. Future applications include surveillance imaging, detection of mural fibrosis, and early assessment of therapy response.
\end{abstract}

\section{Keywords}

MRI; MR enterography; Crohn's disease; ulcerative colitis; imaging

\section{Inflammatory Bowel Disease Overview}

Inflammatory bowel disease (IBD) is a bowel disorder affecting approximately 1.4 million people in North America, with an associated incidence of 20,000-100,000 new cases developing per year((1-3)). Although the precise mechanisms underlying the development of IBD have not been completely elucidated, it is widely accepted that IBD results from an inappropriate response of the gastrointestinal mucosal immune system to gut flora and/or ingested food antigens((1)) IBD patients can be divided into 2 disease forms, Crohn's disease and ulcerative colitis (UC), which differ primarily with respect to histological features and bowel disease distribution((4)). UC is typically confined to the colon and, following surgical resection, intestinal symptoms in UC patients usually resolve. In contrast, Crohn's patients can have disease anywhere in the gastrointestinal tract, making medical therapy the treatment of choice.

Both subtypes of IBD historically have demonstrated a bimodal demographic distribution with a peak incidence occurring during late adolescence and earlier adulthood, followed by a second smaller peak later in adulthood((2-3)). Both forms of IBD are characterized by episodic symptom recurrence over the course of years. UC patients are much more likely to be cured of their abdominal symptoms because intestinal disease involvement is limited to the colon, providing an option for complete surgical excision of diseased bowel in patients refractory to medical therapy. In contrast, Crohn's patients can develop disease anywhere in 
the gastrointestinal tract and often have involvement of multiple discontinuous segments of bowel. As a result, Crohn's disease is more typically characterized by long-term frequent symptomatic recurrence over the lifetime of the patient.

\section{Role Of Imaging In Inflammatory Bowel Disease}

Imaging plays an important role in the evaluation of IBD patients((5-6)). Imaging plays a critical role in the initial diagnosis of disease by providing evidence of the presence, as well as distribution, of abnormal bowel in patients with suspected IBD. The information provided by imaging is combined with clinical, endoscopic, and histological data to provide the diagnosis. Imaging is of particular importance in the evaluation of Crohn's disease to assess the small bowel between the ligament of Treitz and the ileocecal valve, which is not wellevaluated by endoscopy. Cross-sectional imaging modalities (CT, MRI) can also provide information regarding extraluminal disease complications (abscess, fistula, bowel perforation) likely to require more acute intervention, as well as extraintestinal manifestations of IBD that can be symptomatic (primary sclerosing cholangitis, pancreatitis, nephrolithiasis, sacroileitis)((4)). Imaging also provides useful information guiding the treatment of patients with established IBD. Imaging features of the bowel and adjacent mesentery correlate well with clinical indices of disease activity $((5))$ and provide noninvasive determination of the need for therapeutic modulation as well as an indicator of therapy response.

\section{Current Imaging Evaluation Of IBD}

For years, the imaging reference standard for IBD evaluation was barium fluoroscopy. Enteroclysis and small bowel series were used to evaluate the small bowel in Crohn's disease, while barium enema was used to evaluate the colon in Crohn's disease and ulcerative colitis. Barium fluoroscopic evaluation of IBD relied primarily on visualization of abnormalities in bowel mucosal pattern and intestinal caliber((7)). However, fluoroscopic techniques are insensitive for depicting transmural inflammation or the extraluminal complications of inflammatory bowel disease. Over the last decade CT has become the primary imaging modality for evaluating gastrointestinal tract pathology due to its widespread availability, fast scanning time, and ability to produce high-resolution 3dimensional images((8)). CT enterography (CT-E) in particular is tailored to detect bowel wall abnormalities, through the use of large volume neutral enteric contrast and thin slice technique, and has become the preferred CT technique for evaluating inflammatory bowel disease((9)). Currently, routine CT evaluation of Crohn's disease includes assessment of bowel wall thickening, perienteric and pericolonic mesenteric inflammation; lymph node size and number; extraluminal collections (fistulae, abscesses, sinuses); and extraintestinal complications $((10))$. Although CT has proved to be an effective imaging modality for Crohn's disease, one significant limitation is its associated patient exposure to ionizing radiation. The issue of ionizing radiation risk to patients associated with diagnostic radiology examinations has received much attention in recent years; especially for children, in whom the relative cancer mortality risk per unit radiation dose is significantly higher compared with adults((11-12)). This issue is particularly relevant to the IBD population that is often diagnosed at a young age and likely to require frequent imaging over the course of their lifetimes. Epidemiological studies suggest a nonzero radiation-induced cancer risk at exposure levels as low as $75 \mathrm{mSv}$, which are often exceeded in patients diagnosed with Crohn's disease during childhood((13-15)).

\section{Development of MRI For Inflammatory Bowel Disease Evaluation}

Magnetic resonance imaging (MRI) has several intrinsic advantages over other imaging modalities that make it desirable for evaluating inflammatory bowel disease. Foremost of 
these is the lack of ionizing radiation exposure to patients, which is of particular importance to the IBD population that is likely to require numerous imaging studies over the course of their lifetime. Because of the lack of radiation exposure, cinematic MR images can be obtained to examine bowel peristalsis over time. Additional, serial dynamic post-contrast images can be obtained to assess the time course of bowel enhancement. In addition, MRI provides superior soft tissue contrast to $\mathrm{CT}$ in the absence of intravenous contrast, which would be helpful for detecting mesenteric inflammatory changes and bowel wall edema. Historically, MRI of the abdomen has been limited by long acquisition times and extensive motion artifact from respiration. As a result, the initial application of MRI in IBD was pelvic MRI for evaluation of perianal disease in Crohn's disease. The soft tissue contrast of MRI is superior to $\mathrm{CT}$ for delineation of the anal sphincter complex and the pelvis can be imaged in multiple planes with little image degradation from respiratory motion. The last 5-10 years have seen the development of MRI pulse sequences that provide motion-free, high resolution images of the body, which has made MR imaging of the bowel possible((16)). MRI evaluation of the bowel relies predominantly on three sequences((17)). The first is a single shot fast spin echo T2 sequence with half-Fourier reconstruction (e.g. HASTE, SSFSE) that provides motion-free T2 weighted images for evaluating for bowel wall edema and extraluminal fluid collections. The second is balanced steady state free precession (e.g. True FISP, FIESTA) which is exquisitely sensitive to mesenteric changes such as hypervascularity, fibrofatty proliferation, and fistulae. The third is a dynamic fast 3D spoiled gradient echo T1 fat-suppressed post-contrast sequence (e.g. VIBE, LAVA) to evaluate the pattern of bowel enhancement. Other technological innovations leading to effective MR bowel imaging include higher magnetic field strengths (1.5 $\mathrm{T}$ and more recently $3 \mathrm{~T})$, multichannel phased array coils, and parallel image processing (reviewed in ((18))). These technological advances have led to the development and clinical implementation of MRI protocols designed to evaluate the small bowel (MR enterography, enteroclysis) and colon (MR colonography).

\section{Current Roles Of MRI In Inflammatory Bowel Disease Evaluation}

\section{Pelvic MRI For Perianal Disease Evaluation}

Pelvic MRI has become part of the standard imaging workup of patients with Crohn's disease and suspected perianal involvement ((19-20)). The lifetime risk of perianal fistula formation in Crohn's disease ranges from 30-50\%((20)), with the presence of a fistula leading to significant morbidity due to cutaneous drainage or perianal abscess formation. The superior soft tissue contrast of MRI provides detailed anatomic delineation of the anal sphincter complex. It is important to determine the anatomic relationship of perianal fistulae with the internal and external anal sphincters, as well as the levator ani complex (Figure 1), as these can affect the surgical approach and closure technique((21)). Additionally, MRI is sensitive for detection of perianal abscesses requiring urgent intervention.

\section{MRI Evaluation Of Bowel And Enteric Contrast Agents}

MRI evaluation of the bowel typically combines large volume enteric contrast distention of the bowel with dynamic imaging following intravenous contrast administration to increase sensitivity for detecting bowel wall abnormalities((22)). Enteric contrast can be administered either orally (MR enterography) or via nasojejunal tube (MR enteroclysis). Several enteric contrast agents have been used for MR imaging in an attempt to achieve uniform luminal distension with minimal intestinal absorption. Other important considerations for the use of orally administered contrast include patient acceptability and cost((23)). MRI enteric contrast agents are generally classified according to their signal intensity on T1- and T2weighted images((23-24)) and are categorized as positive agents that demonstrate high signal intensity on both T1 and T2 images (e.g. blueberry juice, pineapple juice), negative 
agents (e.g. oral superparamagnetic iron oxide particles) that demonstrate low signal intensity on both T1 and T2 images, and biphasic agents (e.g. water, polyethylene glycol, dilute barium with sorbitol) that demonstrate low signal intensity on T1 and high signal intensity on T2 images. Enteric contrast agents are routinely used for bowel MRI studies in order to displace intraluminal bowel gas that would otherwise cause significant image distortion on gradient echo sequences typically obtained after intravenous contrast administration. Theoretical benefits of low luminal signal intensity on T2 weighted images include better visualization of bowel wall edema and mucosal enhancement as well as discrimination of intraluminal and extraluminal fluid, while positive luminal signal intensity is often favored for the detection of bowel wall thickening. One study examined positive versus negative enteric contrast agents in MR enteroclysis((25)). Positive contrast agents on T2 weighted images were found to be superior for detecting areas of bowel wall thickening. Negative contrast agents were found to be superior for abscess detection, as low intensity intraluminal bowel fluid could be easily distinguished from abscess fluid that remained T2 hyperintense. Biphasic agents have become the predominant oral contrast agents used for bowel MRI, and typically include nonabsorbable high osmolarity additives such as mannitol, polyethylene glycol, and sorbitol to minimize water absorption by bowel.

MR features of signs of active bowel inflammation in inflammatory bowel disease include bowel wall thickening, bowel wall hyperintensity and hyper-enhancement((26)). A layered pattern of bowel wall enhancement consisting of brightly enhancing mucosa (from inflammation) and hypoenhancing submucosa (from edema) has also been shown to be a specific sign of active bowel inflammation (Figure 2)((27-28)). An advantage of MRI over barium fluoroscopic evaluation of IBD is its ability to detect associated mesenteric inflammatory changes suggestive of active disease. Mesenteric features associated with IBD include lymphadenopathy, vasa recta hypervascularity, and fibrofatty infiltration.

\section{MR Enteroclysis}

MR enteroclysis was the first dedicated MRI method for evaluating small bowel in Crohn's disease and was based on fluoroscopic enteroclysis technique (reviewed in ((29))). The enteroclysis technique involves placement of a nasojejunal balloon-tipped catheter under fluoroscopic guidance followed by instillation of a large volume of enteral contrast (1.52.0L) through the catheter, typically using a motorized pump to ensure uniform distention. Balloon inflation minimizes contrast reflux back to the stomach. Large volume enteric contrast distention of the bowel increases sensitivity for detecting areas of abnormal wall thickening or enhancement, which can be missed if the bowel is underdistended ((30)). The instillation of contrast originally was performed in the fluoroscopy suite prior to patient transfer to MRI. However, with the advent of dynamic thick slab MRI techniques, contrast is now routinely instilled under real-time MR guidance until adequate small bowel distention is achieved. Once adequate distention is achieved, multiplanar MR bowel imaging is performed. In some institutions, the patient is placed in the scanner in a prone position to minimize abdominal cavity distention and reduce the number of slices needed to scan the bowel. However, supine positioning may be preferred in some patients who are unable to tolerate scanning in a prone position. In some institutions, intravenous antiperistaltic agents (e.g. glucagon, butylscopolamine) are administered once maximum bowel distention is achieved in order to minimize image degradation from motion as well as to delay enteric contrast clearance. The MR enteroclysis protocol varies by institution by generally involves the single shot T2, balanced steady state, and dynamic T1 fat-suppressed post-contrast sequences described earlier. The coronal plane is used most often for imaging because of the quicker acquisition times relative to the axial plane. The major advantage of MR enteroclysis over conventional fluoroscopic enteroclysis is the ability to demonstrate extraluminal manifestations of IBD. This includes detection of mesenteric inflammatory 
changes such as fibrofatty proliferation and mesenteric hypervascularity, as well as fistulae and abscesses.

\section{MR Enterography}

The MR enterography technique developed as a noninvasive alternative to enteroclysis for small bowel evaluation, analogous to the development of CT enterography, due to the fact that a significant proportion of patients refuse nasojejunal catheter placement. Additionally, MR enteroclysis can be logistically challenging due to the variation in time needed for nasojejunal catheter placement and bowel distention. At high volume MRI centers, this variation can lead to issues with MRI throughput. MR enterography relies upon oral ingestion of contrast by the patient to distend the small bowel; otherwise, the protocol is very similar to MR enteroclysis. Enteric contrast agents for MR enterography tend to be those that are either pleasant tasting or easily flavored [e.g. water, blueberry/pineapple juice, dilute barium with sorbitol (VoLumen, Bracco)].

At our institution, patients receive an oral enteric contrast preparation consisting of $900 \mathrm{~mL}$ of dilute barium with sorbitol (VoLumen) mixed with $300 \mathrm{~mL}$ of ferumoxsil iron oxide suspension (GastroMARK, Tyco). The addition of the iron oxide suspension darkens the lumen on both $\mathrm{T} 2$ and $\mathrm{T} 1$ post-contrast images, which we feel aids in the detection of bowel wall edema and abnormal mucosal enhancement. Oral contrast is ingested continuously over a period of 45 minutes prior to the exam. Patients are scanned in the supine position using a multichannel torso phased array coil. Imaging sequences include coronal and axial singleshot fast spin-echo T2 (SSFSE/HASTE), coronal balanced steady state free precession (FIESTA/True-FISP), axial fast relaxation fast spin-echo T2 (FRFSE/RESTORE) with fat suppression, coronal 3D T1 with fat suppression before and following gadolinium administration $(0.2 \mathrm{mmol} / \mathrm{kg}$ of gadopentetate, Bayer injected at $3 \mathrm{~mL} / \mathrm{sec})$. Dynamic postcontrast images are acquired at 1,3 , and 5 minutes post-contrast. This time period of postcontrast imaging has been shown to be sensitive for visualizing the progressive transmural bowel wall enhancement pattern indicative of active inflammation((28)). Afterward, postcontrast axial 2D fast spoiled-gradient-recalled echo T1 weighted images with fatsuppression are acquired.

A recent prospective study compared MR enterography and MR enteroclysis in Crohn's disease evaluation((31)). This study involved 40 patients with histologically-proven inflammatory bowel disease assigned to undergo either MR enteroclysis or MR enterography. This study demonstrated that MR enteroclysis was superior to MR enterography for bowel distention and detection of mucosal bowel abnormality. The two techniques were comparable for detection of luminal narrowing, mesenteric abnormality, and fistulae. The authors suggest that MR enteroclysis would be the preferred imaging modality for Crohn's disease evaluation but that MR enterography is an acceptable alternative in patients who are unable to tolerate nasojejunal intubation.

\section{Cine MRI Evaluation Of Bowel}

Historically, one radiographic sign of inflammatory bowel disease by fluoroscopic examination has been decreased peristalsis of a segment of bowel. Traditional MRI evaluation of the body has relied upon analysis of static images; however, with the advent of rapid MR imaging techniques, real-time cine MRI evaluation of the bowel is now possible. Most often, the cine sequence used is a coronal thick slab balanced steady state sequence (e.g. FIESTA, True FISP) in which a single volume of the abdomen $10 \mathrm{~mm}$ or thicker is continuously imaged over a period of seconds to evaluate peristaltic motion. One study compared cine MRI using a 17 second balanced steady state dynamic acquisition with conventional MR enterography in 40 patients with established Crohn's disease((32)). The 
cine sequence was used to identify segments of abnormal bowel motility, defined as zones of abnormally increased or decreased peristaltic motion compared with adjacent bowel. The addition of the cine sequence led to detection of an increased number of abnormal bowel segments compared with static MR enterography images alone. The authors believe that alteration in bowel motility is an early imaging sign of Crohn's disease involvement of bowel and helps to identify abnormal bowel segments with subtle signs of inflammation on static images. A significant limitation of this study is the lack of endoscopic or histological verification of imaging findings.

\section{MR Colonography}

MR colonography is a specific MRI technique for evaluating the colon that combines retrograde instillation of water with intravenous contrast and thin section image acquisition (reviewed in((33))). MR colonography was initially applied to imaging surveillance of colorectal cancer, and its use in inflammatory bowel disease was initially reserved for cases where optic colonoscopy was incomplete due to technical difficulty or patient intolerance. However, MR colonography does offer some advantages over endoscopy including evaluation of submucosal and mesenteric involvement, and its noninvasive nature means there is no risk of colonic perforation in patients presenting with acute symptoms. Prior to MR colonography, patients typically undergo a bowel cleansing regimen similar to that used for colonoscopy. Unlike CT colonography which uses colonic insufflation with air, MR colonography typically involves colonic distension via warm water enema to avoid susceptibility artifacts that could degrade image quality. MR colonographic features of inflammatory bowel disease are similar to those seen on MR enterography, including bowel wall thickening and edema, mural hyperenhancement, lymphadenopathy, and mesenteric inflammatory changes.

An additional potential benefit of MR colonography is in helping to distinguish between Crohn's disease and ulcerative colitis in cases of indeterminate colitis. In these cases, MRI is advantageous for detecting extraluminal disease manifestations such as fistulae and abscesses that are more suggestive of Crohn's disease. Additionally, because MR colonography also images the small bowel, detection of small bowel disease also indicates Crohn's disease rather than ulcerative colitis. A 2005 study of 22 patients with known or suspected inflammatory bowel disease who underwent MR colonography with positive rectal contrast (gadolinium/water mixture) followed immediately by optical colonoscopy((34)). Compared with endoscopic reference, MR colonographic sensitivity for detecting colonic involvement of IBD on a per bowel segment basis was $31.6 \%$ for Crohn's disease and 58.8\% for ulcerative colitis. The authors concluded from this study that MR colonography was not suitable for assessing the extent of colonic inflammation in inflammatory bowel disease. A second study from the same year evaluated 15 normal subjects and 23 subjects with suspected IBD by MR colonography(35) to detect colonic inflammation, using endoscopic biopsy as reference. MR colonographic assessment of colonic inflammation (based on abnormal enhancement, wall thickening, lymphadenopathy, and loss of haustral folds) was shown to be $87 \%$ sensitive and $100 \%$ specific compared with histologic standard. Based on this data, MR colonography is considered a promising noninvasive method for monitoring IBD activity and therapeutic efficacy.

\section{Comparison Of MRI With Other Imaging Modalities}

The MRI protocol most frequently compared with other modalities has been MR enterography. A number of recent prospective studies have demonstrated MR enterography to be at least comparable to other imaging modalities for detection of small bowel disease in Crohn's patients. One study comparing contrast-enhanced MRI and CT performed on different days in adult Crohn's disease patients demonstrated MRI to be superior for 
detection of subtle bowel inflammatory changes((36)). Another study in 2005 compared MR enterography with fluoroscopic barium small bowel series(37). In this study, 30 subjects with established Crohn's disease referred for barium small bowel series also underwent MR enterography using dilute barium within 28 days to assess for concordance of the two imaging modalities. In 18 subjects the two modalities demonstrated similar Crohn's imaging features ( 10 of the subjects were normal). Among the 12 subjects with discordant results, SBFT demonstrated additional strictures or fistulae in 4 , while MRI demonstrated additional information in 8 by identifying active inflammation in stricture areas (based on abnormal wall enhancement or mesenteric inflammatory changes). No endoscopic or histologic validation was included in this study. A 2009 study examined 30 patients with established Crohn's disease who underwent MR enterography and CT enterography the same day, followed by barium SBFT and ileocolonoscopy within 1 week(38). A 1\% sorbitol oral contrast solution was used for both CT and MRI. Receiver operator curve analysis demonstrated all three imaging modalities to be similar for detection of terminal ileum active inflammation using endoscopic evaluation as the reference standard (area under curve values ranging from 0.88-0.95). CT and MRI both demonstrated superior accuracy to SBFT for detection of extraenteric Crohn's complications including fistulae, sinus tracts, and abcesses, when compared with physical exam or surgical reference. Both CT and MRI also demonstrated more segments of active small bowel inflammation proximal to the terminal ileum than SBFT, although the results were not statistically significant. A second study from 2009 prospectively compared MR enterography and CT enterography performed the same day on 30 patients with suspected Crohn's disease, using ileocolonoscopic findings performed within 30 days as reference standard. This study demonstrated the two modalities to have comparable sensitivity (MRI 90.5\%, CT 95.2\%) for detecting active small bowel inflammation in adults with Crohn's disease((39)).

Wireless capsule endoscopy (WCE) is another minimally invasive technique that has been developed recently for small bowel evaluation(40). This technique involves a video capsule endoscope that is swallowed and propelled through the gastrointestinal tract by peristalsis. The endoscope captures images of the small bowel that are transmitted to aerials taped on the body and then stored on a portable recorder. This technique provides endoluminal evaluation of the entire small bowel. Advantages of this technique over MRI include the ability to detect subtle mucosal abnormalities. Disadvantages include potential inability to pass the capsule endoscope in patients with small bowel strictures, as well as inability of WCE to visualize extraluminal complications of Crohn's disease. Very few studies have directly compared WCE to MRI for small bowel evaluation. One study(41) compared WCE with MR enteroclysis in patients with suspected small bowel disease. A total of 17 patients with known or suspected Crohn's disease were imaged. WCE depicted a higher number of inflammatory lesions in the jejunum, and proximal ileum compared with MR enteroclysis, while the two modalities demonstrated a similar number of inflammatory lesions in the terminal ileum. This study did not include any histologic or endoscopic validation of findings.

\section{New And Emerging Roles Of MRI In Inflammatory Bowel Disease}

MRI has been shown to be sensitive for detecting certain aspects of Crohn's disease such as small bowel inflammation, perianal fistulae and abscesses. Recent technical advances in body MRI, including higher magnet field strength, parallel image processing, and motion artifact reduction techniques, should lead to shorter scan times and increased spatial resolution for detecting subtle inflammatory changes((18)). Such advances should make it possible for MRI to replace CT as the primary imaging modality for Crohn's disease patients in the near future. Such surveillance imaging would extend the role of MRI beyond its current indications to include detection of colonic inflammatory changes, as well as 
extraluminal imaging features of disease such as mesenteric lymphadenopathy, ascites, and fibrofatty proliferative changes. The incorporation of MRI into routine Crohn's disease surveillance would lead to a significant reduction in patient lifetime radiation exposure, that majority of which currently derives from $\mathrm{CT}((14))$.

Another future role for MRI in Crohn's disease is the detection of mural fibrosis. Most studies examining the accuracy of MRI for assessing Crohn's disease activity focus on the distinction between active and inactive inflammation $((26,39))$, with the presence of active disease considered to be an indication to initiate or modify medical therapy. A complementary approach would be to take advantage of the soft tissue contrast of MRI to detect mural fibrosis, which would helpful for selecting patients likely to require surgical bowel resection. Fibrosis is considered a late-phase irreversible result of chronic bowel wall inflammation leading to collagen fiber deposition in the submucosal and serosal layers of the bowel wall. Mural fibrosis frequently leads to luminal narrowing associated with proximal bowel obstruction and, unlike acute inflammatory strictures, fibrotic strictures usually require surgical resection to alleviate the associated obstruction. Reported MRI findings associated with mural fibrosis include bowel wall T2 hypointensity((42)) and lack of enhancement((27)). Early detection of mural fibrosis in Crohn's disease patients potentially would be useful to facilitate surgical resection of irrevocably diseased bowel segments, thereby reducing the number of symptomatic recurrences.

MRI protocols have been developed successfully for evaluation of the small bowel (MR enterography) or the colon (MR colonography). A future challenge would be the development of a single combined protocol for evaluation of both small and large bowel. Such a protocol would be particularly useful in the Crohn's population, which can develop areas of bowel inflammation anywhere in the gastrointestinal tract. A 2005 study((35)) combined MR enterography with $1.5 \mathrm{~L}$ of oral contrast and rectal distention via 500-1000 $\mathrm{mL}$ water enema in 20 patients with known Crohn's disease and compared it with 20 patients who underwent MR enterography without rectal distention. Comparison of MRI findings was made with colonoscopy performed within 7 days of the MRI. The addition of rectal water was associated with improved distention of both the terminal ileum and the rectum compared with oral contrast alone. Diagnostic accuracy for inflammation of both the terminal ileum and the colon was improved with rectal enema administration. One issue with such combined enteric contrast administration is patient compliance. Large volume oral contrast administration alone makes some IBD patients distended and uncomfortable, and the addition of rectal contrast is likely to be less well-accepted. An enteric contrast regimen leading to distention of small and large bowel by oral administration alone would be ideal.

A final emerging application of MRI in inflammatory bowel disease would be its use as a biomarker of therapeutic response. Treatment of inflammatory bowel disease has been revolutionized due to the recent introduction of biologic therapies targeting molecular pathways thought to contribute to bowel inflammation, such as the proinflammatory cytokine TNF- $\alpha$, lymphocyte signaling molecules CTLA- 4 and CD20, and the $\alpha_{4}$ integrin adhesion molecule mediating leukocyte migration((43-44)). These agents are generally considered to be more specific for IBD compared with traditional corticosteroids or immunomodulatory agents. Indications for biologic agents include patients with inflammatory bowel disease refractory to standard therapies, patients who are steroid dependent, and patients with draining fistulae or systemic extraintestinal disease manifestations. Most of the biologic agents currently in clinical practice are either recombinant peptides or chimeric antibodies, which are more expensive to produce compared with traditional compound-based drugs((44)), meaning that therapy with these agents can be associated with high financial costs to the patients and the healthcare system as a whole. An early noninvasive assessment of treatment response potentially would be of 
great financial benefit to patients undergoing biologic therapy, by ensuring that patients who are refractory to treatment do not remain on medication for longer than necessary.

Additionally, biologic agents have their own unique side effect profile including increased risk of serious infections, as well as rarer side effects such as neurologic disorders, CHF, and hematologic malignancies. Much recent attention has been focused on cases of hepatosplenic $\mathrm{T}$ cell lymphoma observed in young adult IBD patients treated with a combination of biologic and immunomodulatory agents((45)). An early imaging assessment of treatment response or failure would also be beneficial to minimize potential side effects associated with unnecessary prolonged treatment. MRI is particularly well-suited to serve as an imaging biomarker of therapeutic efficacy because of its lack of ionizing radiation, which makes it an ideal modality for repeated assessment before and during treatment.

\section{CONCLUSION}

The role of MRI in the assessment of inflammatory bowel disease continues to expand due to its lack of ionizing radiation exposure and superior soft tissue contrast. MRI currently is the modality of choice for detecting perianal inflammation and fistulae, as well as extraintestinal disease manifestations. Recent evidence suggests a role for MRI in the detection of active small bowel inflammation in patients with known IBD. Other potential roles for MRI in IBD evaluation include detection of mural fibrosis and early assessment of treatment response. As the spatial resolution and scanning time of MRI continue to improve as a result of technical innovation, MRI will likely also prove to be suitable as the primary modality for IBD imaging surveillance.

\section{Acknowledgments}

The authors thank Dr. Katherine Nimkin for her assistance with creation of MRI protocols for evaluating inflammatory bowel disease.

Grant support: This work is supported by a Catalyst award from the National Institutes of Health and Harvard Medical School (UL1 RR025758-02 to M.S.G. and M.G.H.)

\section{References}

1. Baumgart DC, Carding SR. Inflammatory bowel disease: cause and immunobiology. Lancet. 2007; 369(9573):1627-1640. [PubMed: 17499605]

2. Friedman, S.; Blumberg, RS. Inflammatory Bowel Disease. In: Braunwald, E.; Fauci, AS.; Kasper, DL.; Hauser, SL.; Longo, DL.; Jameson, JL., editors. Harrison's Principles of Internal Medicine. 15. New York: McGraw-Hill; 2001. p. 1679-1692.

3. Loftus EV Jr. Clinical epidemiology of inflammatory bowel disease: Incidence, prevalence, and environmental influences. Gastroenterology. 2004; 126(6):1504-1517. [PubMed: 15168363]

4. Baumgart DC, Sandborn WJ. Inflammatory bowel disease: clinical aspects and established and evolving therapies. Lancet. 2007; 369(9573):1641-1657. [PubMed: 17499606]

5. Patak MA, Mortele KJ, Ros PR. Multidetector row CT of the small bowel. Radiol Clin North Am. 2005; 43(6):1063-1077. viii. [PubMed: 16253662]

6. Mackalski BA, Bernstein CN. New diagnostic imaging tools for inflammatory bowel disease. Gut. 2006; 55(5):733-741. [PubMed: 16609136]

7. Carucci LR, Levine MS. Radiographic imaging of inflammatory bowel disease. Gastroenterol Clin North Am. 2002; 31(1):93-117. ix. [PubMed: 12122746]

8. Horton KM, Fishman EK. The current status of multidetector row CT and three-dimensional imaging of the small bowel. Radiol Clin North Am. 2003; 41(2):199-212. [PubMed: 12659334]

9. Paulsen SR, Huprich JE, Fletcher JG, et al. CT enterography as a diagnostic tool in evaluating small bowel disorders: review of clinical experience with over 700 cases. Radiographics. 2006; 26(3): 641-657. discussion 657-662. [PubMed: 16702444] 
10. Gore RM, Balthazar EJ, Ghahremani GG, Miller FH. CT features of ulcerative colitis and Crohn's disease. AJR Am J Roentgenol. 1996; 167(1):3-15. [PubMed: 8659415]

11. Brenner DJ, Elliston CD, Hall EJ, Berdon WE. Estimates of the cancer risks from pediatric CT radiation are not merely theoretical: comment on "point/counterpoint: in x-ray computed tomography, technique factors should be selected appropriate to patient size. against the proposition". Med Phys. 2001; 28(11):2387-2388. [PubMed: 11764047]

12. Rice HE, Frush DP, Farmer D, Waldhausen JH. Review of radiation risks from computed tomography: essentials for the pediatric surgeon. J Pediatr Surg. 2007; 42(4):603-607. [PubMed: 17448753]

13. Brenner DJ. Should computed tomography be the modality of choice for imaging Crohn's disease in children? The radiation risk perspective. Gut. 2008; 57(11):1489-1490. [PubMed: 18941001]

14. Desmond AN, O'Regan K, Curran C, et al. Crohn's disease: factors associated with exposure to high levels of diagnostic radiation. Gut. 2008; 57(11):1524-1529. [PubMed: 18443021]

15. Pierce DA, Preston DL. Radiation-related cancer risks at low doses among atomic bomb survivors. Radiat Res. 2000; 154(2):178-186. [PubMed: 10931690]

16. Furukawa A, Saotome T, Yamasaki M, et al. Cross-sectional imaging in Crohn disease. Radiographics. 2004; 24(3):689-702. [PubMed: 15143222]

17. Gourtsoyiannis NC, Papanikolaou N, Karantanas A. Magnetic resonance imaging evaluation of small intestinal Crohn's disease. Best Pract Res Clin Gastroenterol. 2006; 20(1):137-156. [PubMed: 16473805]

18. MacKenzie JD, Vasanawala SS. Advances in pediatric MR imaging. Magn Reson Imaging Clin N Am. 2008; 16(3):385-402. v. [PubMed: 18585595]

19. Koelbel G, Schmiedl U, Majer MC, et al. Diagnosis of fistulae and sinus tracts in patients with Crohn disease: value of MR imaging. AJR Am J Roentgenol. 1989; 152(5):999-1003. [PubMed: 2705359]

20. Szurowska E, Wypych J, Izycka-Swieszewska E. Perianal fistulas in Crohn's disease: MRI diagnosis and surgical planning: MRI in fistulazing perianal Crohn's disease. Abdom Imaging. 2007

21. Halligan S, Buchanan G. MR imaging of fistula-in-ano. Eur J Radiol. 2003; 47(2):98-107. [PubMed: 12880990]

22. Fidler J. MR imaging of the small bowel. Radiol Clin North Am. 2007; 45(2):317-331. [PubMed: 17502220]

23. Laghi A, Paolantonio P, Iafrate F, Altomari F, Miglio C, Passariello R. Oral contrast agents for magnetic resonance imaging of the bowel. Top Magn Reson Imaging. 2002; 13(6):389-396. [PubMed: 12478019]

24. Fidler JL, Guimaraes L, Einstein DM. MR imaging of the small bowel. Radiographics. 2009; 29(6):1811-1825. [PubMed: 19959523]

25. Rieber A, Aschoff A, Nussle K, et al. MRI in the diagnosis of small bowel disease: use of positive and negative oral contrast media in combination with enteroclysis. Eur Radiol. 2000; 10(9):13771382. [PubMed: 10997423]

26. Maccioni F, Viscido A, Broglia L, et al. Evaluation of Crohn disease activity with magnetic resonance imaging. Abdom Imaging. 2000; 25(3):219-228. [PubMed: 10823437]

27. Koh DM, Miao Y, Chinn RJ, et al. MR imaging evaluation of the activity of Crohn's disease. AJR Am J Roentgenol. 2001; 177(6):1325-1332. [PubMed: 11717076]

28. Del Vescovo R, Sansoni I, Caviglia R, et al. Dynamic contrast enhanced magnetic resonance imaging of the terminal ileum: differentiation of activity of Crohn's disease. Abdom Imaging. 2007

29. Prassopoulos P, Papanikolaou N, Grammatikakis J, Rousomoustakaki M, Maris T, Gourtsoyiannis N. MR enteroclysis imaging of Crohn disease. Radiographics. 2001; 21(Spec No):S161-172. [PubMed: 11598255]

30. Low RN, Francis IR. MR imaging of the gastrointestinal tract with i.v. gadolinium and diluted barium oral contrast media compared with unenhanced MR imaging and CT. AJR Am J Roentgenol. 1997; 169(4):1051-1059. [PubMed: 9308464] 
31. Masselli G, Casciani E, Polettini E, Gualdi G. Comparison of MR enteroclysis with MR enterography and conventional enteroclysis in patients with Crohn's disease. Eur Radiol. 2008; 18(3):438-447. [PubMed: 17899102]

32. Froehlich JM, Waldherr C, Stoupis C, Erturk SM, Patak MA. MR motility imaging in Crohn's disease improves lesion detection compared with standard MR imaging. Eur Radiol. 2010; 20(8): 1945-1951. [PubMed: 20379822]

33. Rimola J, Rodriguez S, Garcia-Bosch O, et al. Role of 3.0-T MR colonography in the evaluation of inflammatory bowel disease. Radiographics. 2009; 29(3):701-719. [PubMed: 19448111]

34. Schreyer AG, Rath HC, Kikinis R, et al. Comparison of magnetic resonance imaging colonography with conventional colonoscopy for the assessment of intestinal inflammation in patients with inflammatory bowel disease: a feasibility study. Gut. 2005; 54(2):250-256. [PubMed: 15647190]

35. Ajaj W, Lauenstein TC, Langhorst J, et al. Small bowel hydro-MR imaging for optimized ileocecal distension in Crohn's disease: should an additional rectal enema filling be performed? J Magn Reson Imaging. 2005; 22(1):92-100. [PubMed: 15971189]

36. Low RN, Francis IR, Politoske D, Bennett M. Crohn's disease evaluation: comparison of contrastenhanced MR imaging and single-phase helical CT scanning. J Magn Reson Imaging. 2000; 11(2): 127-135. [PubMed: 10713944]

37. Bernstein CN, Greenberg H, Boult I, Chubey S, Leblanc C, Ryner L. A prospective comparison study of MRI versus small bowel follow-through in recurrent Crohn's disease. Am J Gastroenterol. 2005; 100(11):2493-2502. [PubMed: 16279905]

38. Lee SS, Kim AY, Yang SK, et al. Crohn disease of the small bowel: comparison of CT enterography, MR enterography, and small-bowel follow-through as diagnostic techniques. Radiology. 2009; 251(3):751-761. [PubMed: 19276325]

39. Siddiki HA, Fidler JL, Fletcher JG, et al. Prospective comparison of state-of-the-art MR enterography and CT enterography in small-bowel Crohn's disease. AJR Am J Roentgenol. 2009; 193(1):113-121. [PubMed: 19542402]

40. Iddan G, Meron G, Glukhovsky A, Swain P. Wireless capsule endoscopy. Nature. 2000; 405(6785):417. [PubMed: 10839527]

41. Golder SK, Schreyer AG, Endlicher E, et al. Comparison of capsule endoscopy and magnetic resonance (MR) enteroclysis in suspected small bowel disease. Int J Colorectal Dis. 2006; 21(2): 97-104. [PubMed: 15846497]

42. Maglinte DD, Gourtsoyiannis N, Rex D, Howard TJ, Kelvin FM. Classification of small bowel Crohn's subtypes based on multimodality imaging. Radiol Clin North Am. 2003; 41(2):285-303. [PubMed: 12659339]

43. Clark M, Colombel JF, Feagan BC, et al. American gastroenterological association consensus development conference on the use of biologics in the treatment of inflammatory bowel disease, June 21-23, 2006. Gastroenterology. 2007; 133(1):312-339. [PubMed: 17631151]

44. Bosani M, Ardizzone S, Porro GB. Biologic targeting in the treatment of inflammatory bowel diseases. Biologics. 2009; 3:77-97. [PubMed: 19707398]

45. Cucchiara S, Escher JC, Hildebrand H, Amil-Dias J, Stronati L, Ruemmele FM. Pediatric inflammatory bowel diseases and the risk of lymphoma: should we revise our treatment strategies? J Pediatr Gastroenterol Nutr. 2009; 48(3):257-267. [PubMed: 19274777] 

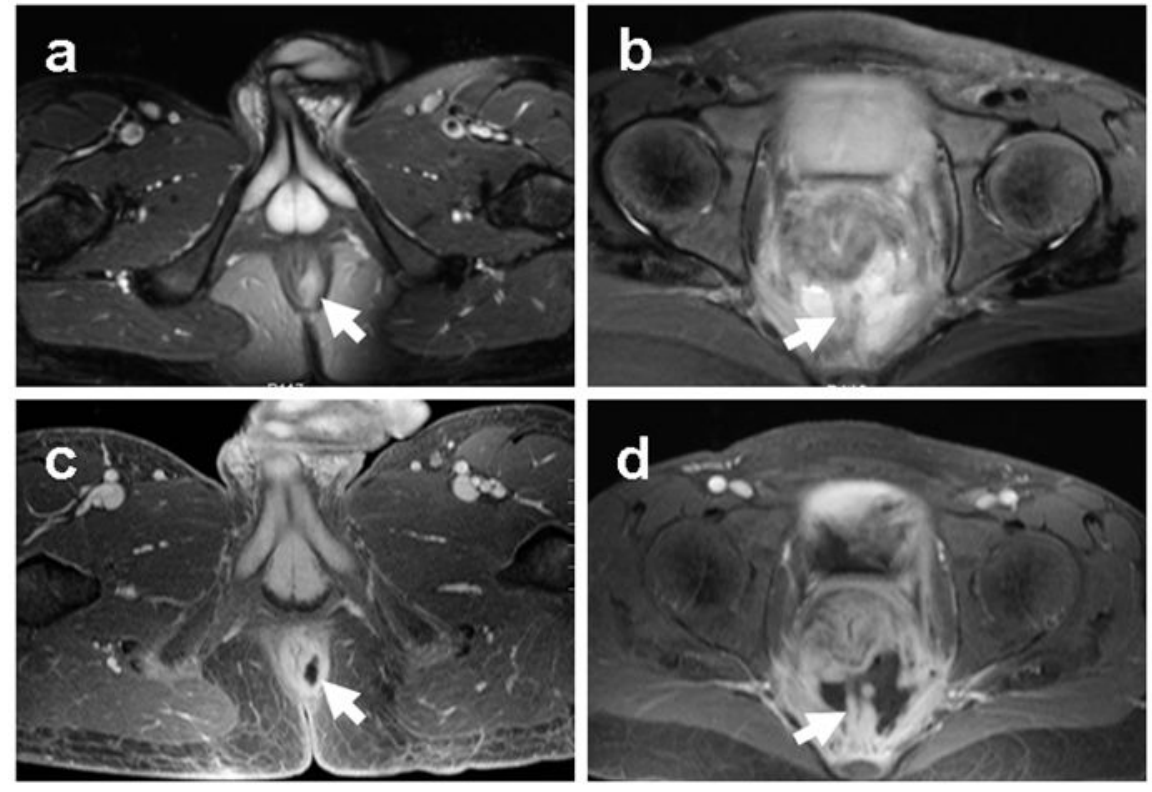

Figure 1.

Pelvic MRI of IBD perianal disease. Representative axial T2 fat suppressed (a, b) and postcontrast T1 fat-suppressed (c, d) images demonstrating an intersphincteric perianal fistula (a, c) and presacral abscess (b, d) in two patients with known Crohn's disease. Arrows indicate sites of disease. 

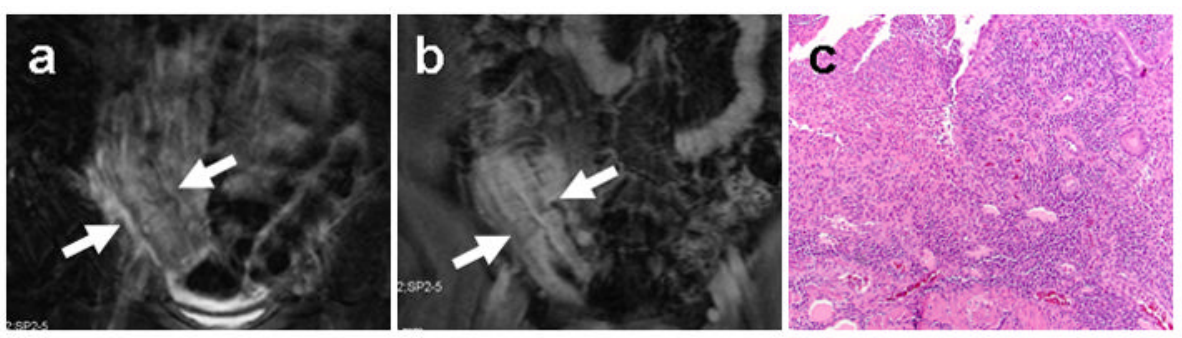

Figure 2.

MR enterographic detection of active bowel inflammation. Coronal T2 fat-suppressed (a) and post-contrast $\mathrm{T} 1$ fat-suppressed (b) images from an MR enterography study on a Crohn's disease patient demonstrate an area of thickened terminal ileum (arrows) exhibiting T2 hyperintensity and layered enhancement consistent with active disease. Corresponding surgical bowel excision specimen from the same patient (c) demonstrates neutrophilic invasion of mucosal crypts consistent with active inflammatory changes. 


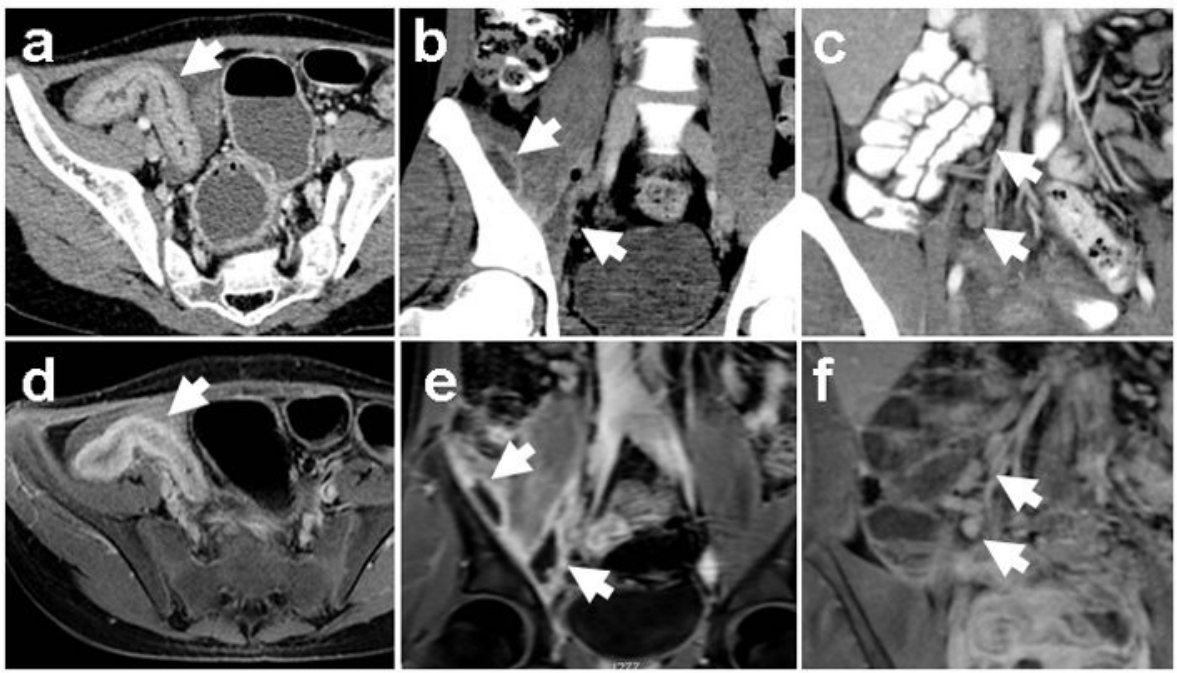

Figure 3.

MRI detection of IBD imaging features. Corresponding image pairs from contrast enhanced CT (top row) and MR enterography (bottom row) studies on the same patients with known IBD demonstrate bowel wall thickening (a, d), intramuscular abscesses (b, e), and mesenteric lymphadenopathy (c, f). Arrows indicate the abnormalities. 